\title{
Auditory Localization Performances in Subjects with Simulated Unilateral Conductive Hearing Loss
}

\author{
Mohd Normani Zakaria ${ }^{1}$, Wan Najibah Wan Mohamad ${ }^{1}$, \\ Noor Alaudin Abdul Wahab², and Nashrah Maamor ${ }^{2}$ \\ ${ }^{1}$ Audiology Programme, School of Health Sciences, Universiti Sains Malaysia, Kelantan, Malaysia \\ ${ }^{2}$ Audiology Programme, Faculty of Health Sciences, Universiti Kebangsaan Malaysia, Kuala Lumpur, Malaysia
}

\section{Dear Editor,}

We read the article considerately written by Parisa and colleagues [1] entitled "Horizontal Localization in Simulated Unilateral Hearing Loss" with great interest. In this study, the sound localization ability in the horizontal plane was tested in healthy adults with and without the use of earplug. The authors should be commended for their research effort, particularly with the use of multiple frequencies to understand sound localization deficits in subjects with simulated unilateral conductive hearing loss. Nevertheless, we wish to highlight several issues worthy of consideration.

In the first part of study, the threshold shifts (produced by the use of binaural earplugs) were compared between the tested frequencies. Higher thresholds shifts were noted at high frequencies, which are sensible. These data support the efficiency of the chosen earplugs in achieving the desired sound attenuation levels so that optimum results would be obtained in the subsequent localization task.

In the second part of study, auditory localization performances for specific locations were compared between two test conditions (with earplug vs. without earplug), as well as between the test frequencies. The subjects' perceived locations were documented and analyzed as intended. However, we feel that the clarity of results presentation can be further refined. In this respect, the addition of localization error results can be

Received August 31, 2018 / Accepted October 19, 2018

Address for correspondence

Mohd Normani Zakaria, PhD

Audiology Programme, School of Health Sciences, Universiti Sains

Malaysia, 16150 Kubang Kerian, Kelantan, Malaysia

Tel +60-9-7677691 / Fax +60-9-7677515 / E-mail mdnorman@usm.my

This is an Open Access article distributed under the terms of the Creative Commons Attribution Non-Commercial License (https://creativecommons.org/licenses/by-nc/4.0/) which permits unrestricted non-commercial use, distribution, and reproduction in any medium, provided the original work is properly cited. beneficial. For example, as reported by the authors in the results section, "The results showed that for all frequencies at +30 and +60 degrees, the localization azimuth in the occluded right ear situation is significantly less than the non-occluded situation ( $p<0.001)$." Herein, the word "less" can be confusing (does it mean better or worse result?), perhaps because only the perceived location results were shown (which were mostly "negative"). If localization errors are included (and followed by appropriate inferential statistics), the localization performances can be conveniently compared between the test conditions. As such, the localization errors would be much higher in the occluded condition than in the non-occluded condition for each sound location (indicating the "loss" of interaural level difference cue when the ear is occluded). Nevertheless, the authors did include some localization error results in the discussion section so that comparisons could be made with the previous studies (supporting the relevancy of having localization error analyses).

Additionally, another essential information that was not stated in the paper is the duration of stimuli used. Since the subjects were blindfolded and instructed to move their head to indicate the perceived location, the duration of stimuli can be a critical factor. Too short stimulus duration would produce "unnecessary" localization errors, whereas too long stimulus duration may not give "genuine" results as additional cues (eye and head movements) may contribute resulting in better localization performances [2-4]. Nonetheless, the authors are to be congratulated for their effort in studying this fundamental aspect of hearing and their study outcomes are indeed consistent with the previous auditory localization studies $[5,6]$.

\section{Conflicts of interest}

The authors have no financial conflicts of interest. 


\section{REFERENCES}

1) Parisa A, Reza NA, Jalal SS, Mohammad K, Homa ZK. Horizontal localization in simulated unilateral hearing loss. J Audiol Otol 2018; 22:39-44

2) Pollack I, Rose M. Effect of head movement on the localization of sounds in the equatorial plane. Percept Psychophys 1967;2:591-6.

3) Perrott DR, Ambarsoom H, Tucker J. Changes in head position as a measure of auditory localization performance: auditory psychomotor coordination under monaural and binaural listening conditions. J Acoust Soc Am 1987;82:1637-45.

4) Frens MA, Van Opstal AJ. A quantitative study of auditory-evoked saccadic eye movements in two dimensions. Exp Brain Res 1995;107: 103-17.

5) Humes LE, Allen SK, Bess FH. Horizontal sound localization skills of unilaterally hearing-impaired children. Audiology 1980;19:508-18.

6) Gustafson TJ, Hamill TA. Differences in localization ability in cases of right versus left unilateral simulated conductive hearing loss. J Am Acad Audiol 1995;6:124-8.

\section{Response}

\section{Anvarsamarein Parisa ${ }^{1}$, Nazeri Ahmad Reza ${ }^{1}$ Sameni Seyyed Jalal ${ }^{2}$, Kamali Mohammad ${ }^{3}$, and Zarrin Koob Homa ${ }^{1}$}

${ }^{1}$ Department of Audiology, School of Rehabilitation Sciences, Shahid Beheshti Medical University, Tehran, Iran

${ }^{2}$ Departments of Audiology, ${ }^{3}$ Basic Sciences in Rehabilitation, School of Rehabilitation Sciences,

Iran University of Medical Sciences, Tehran, Iran

To the Editor:

All authors have appreciate your thoughtful comment on our article published in the Journal of Audiology and Otology. We make brief comment on your queries.

First, the authors have confirmed our results about the threshold shifts produced by use of binaural earplugs. Second, regarding the calculation of localization error, we will try to apply it in our upcoming studies. Third, considering the duration of test signals is a noteworthy point. As we used the usual clinical audiometer, we tried to keep the duration in a constant manner (around $1 \mathrm{sec}$ ). However, controlling the duration by suitable software seems to be more accurate and will be mentioned in next studies.

We believe that your comments have enriched our study and deeply appreciate your attention. 